\title{
Influence of surface integrity on the plastic flow in analogous testing of $42 \mathrm{CrMo} 4$
}

\author{
Rafael Hild ${ }^{l, *}$, Andreas Feuerhack ${ }^{1}$, Daniel Trauth ${ }^{1}$, Dennis C. Hoffmann ${ }^{2}$, Nathan C. Kruppe ${ }^{2}$, Tobias Brögelmann ${ }^{2}$, \\ Kirsten Bobzin ${ }^{2}$ and Fritz Klocke ${ }^{l}$
}

${ }^{1}$ Laboratory for Machine Tools and Production Engineering (WZL), RWTH Aachen University

${ }^{2}$ Surface Engineering Institute (IOT), RWTH Aachen University

\begin{abstract}
Surface modification of workpieces for cold forming processes is common in industrial practice. Specific surface topographies manufactured by means of shot peening form lubricant pockets, which provide a continuous lubricating film throughout the process. Furthermore, hydrostatic as well as hydrodynamic lubrication effects emerge, which further reduce the friction during the process and protect the tool from wear effects. These effects are well researched and applied in order to enable cold forming processes. In dry forming processes, no lubricants are used, and the positive lubrication effects do not appear. Surface modifications by shot peening or knurling change surface integrity in terms of hardness, residual stresses and geometry. The effects of the changed surface integrity on the forming process are not researched. In tribometer tests, a hard and rough surface texture lead to a lower frictional shear stress, whereas a rather smooth and hard surface texture leads to a lower punch force in an extrusion process. The mechanisms between surface textures and the smoothing and forming process are unknown. Furthermore, findings of the tribometer tests cannot be fully transferred to the extrusion process. As in tribometer tests a pin is used as tool and in extrusion a whole die, a discrepancy between the most suitable surface texture for a friction reduction results out of different flow conditions. Still, the tribometer tests give a first reference on a surface pretreament. The paper deals with an investigation of surface textures in order to distinguish characteristics of surface textures respectively a friction reduction. By means of indentation tests of the quenched and tempered steel $42 \mathrm{CrMo} 4$ the plastic flow in dependency of different surface integrities are researched. The results show which characteristics help to lower the punch force the most, so for further investigations an optimized surface modification is set up in order to enable a dry metal forming process.
\end{abstract}

Keywords: Cold forming, Tribology, Surface integrity

\section{Introduction}

Cold forming processes are highly effective due to a high ratio of material utilization, high product quality and short production cycles. At the same time, cold forming processes are highly reproducible and suitable for the production of high quantities [1]. Nowadays, lubricants in combination with surface texturing of workpieces are used to enable cold forming processes [2]. Lubricants reduce process loads and prevent wear [3]. By means of surface texturing like shot peening a surface enlargement is achieved [4]. The surface enlargement supports a higher lubricant capacity and at the same time provides a lubricant film throughout the forming process by means of lubricant pockets [4]. In order to raise the process productivity of cold forming even further and at the same time reduce ecological problems of lubricants, cold forging without lubricants is investigated [5]. Cold forging without lubricants would reduce process steps as applying and removing of lubricants as well as recycling steps [5]. Due to the absence of lubricants the process loads may increase substantially and could cause damage to the tools or deteriorate the process [6].

There is some research on dry metal forming regarding sheet metal forming. By means of DLCcoatings on deep drawing tools, a dry deep drawing process was realized by Murakawa [6]. Murakawa et al. extended this research by a review of different tribological conditions and found non-silicon films most suitable [7]. Osakada et al. investigated the impact of tool surface roughness on the coefficient of friction in dry sheet metal forming and found that a higher roughness resulted in a higher coefficient of friction [8]. Kataoka et al. made researches regarding ceramic tools and achieved comparable results to the lubricated sheet metal forming of low-alloyed steels [9]. Tamaoki et al. developed electroconductive ceramic tools for deep drawing [10] and was able to reach drawn quantities as high as with

* Corresponding author: r.hild@wzl.rwth-aachen.de 
lubricants [11]. Due to higher process loads and surface extension a transfer of these findings to cold metal forming is not directly possible [12]. Furthermore, the investigation of the influence of surface textures on cold forging processes are rather rudimental. Wang et al. investigated surface texturing of billets in upsetting and ironing processes and found a higher lubricant capacity for textured surfaces. An investigation on the dependency of lubricant capacity or forming force reduction in favor or the surface roughness or hardness was not done [13]. Groche et al. investigated the influence of surface textures on forming tools in order to reduce friction and wear. It was possible to reduce the friction ratio up to $40 \%$, but an investigation of a surface textured workpiece was not done [14].

The aim of the authors' work is to investigate the surface textures of workpieces and their influence on the forming capability and friction reduction. The understanding of the mechanisms between a textured workpiece and the forming tool helps to enable a dry metal forming process, see Fig. 1 [13]. In former investigation the influence of surface textured workpieces on the use of self-lubricating PVD-coated tools is done [19]. The boundary conditions of the tribosystem are either changed by the use of a self-lubricating coating $(\mathrm{Cr}, \mathrm{Al}) \mathrm{N}+\mathrm{X}: \mathrm{S}(\mathrm{X}=\mathrm{Mo}, \mathrm{W})$ on tools or surface textures by means of shot peening. In this work, former experimental and numerical researches are extended with an analysis of surface textures and their forming behavior. The paper demonstrates how a dry metal forming process using surface textures on workpieces and self-lubricating tool coatings can be realized, see Fig. 1 .

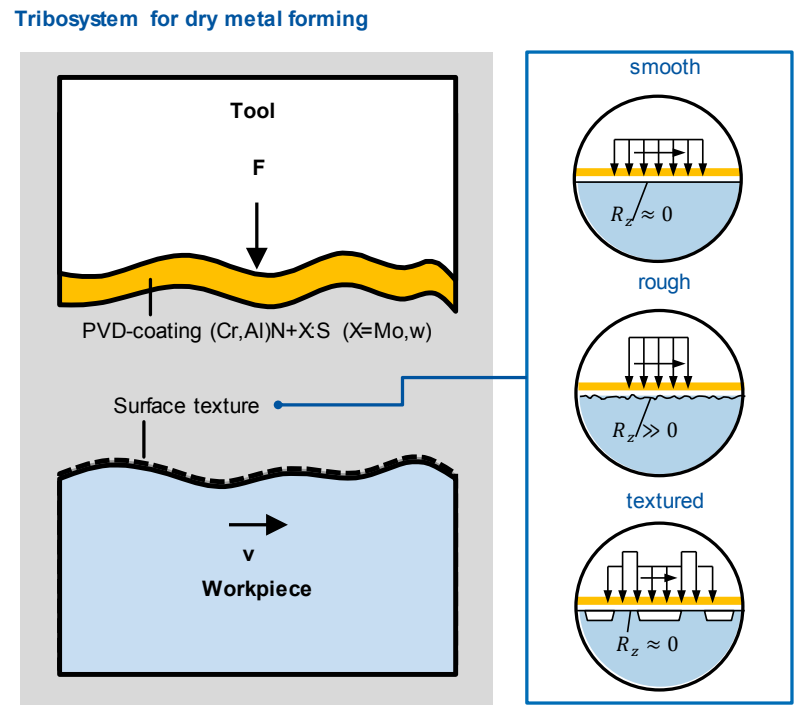

Fig 1. Research objectives for a novel tribosystem for dry metal forming in view of the tool from the surface engineering view (IOT) and surface texturing view (WZL) [13]

\section{Previous Work}

The current research is realized in the second phase of the priority program SPP 1676 of the German Research Foundation. In phase I, new approaches to enable dry metal forming processes were investigated initially using stationary loads. In this section an explanation of a novel pin-on-cylinder tribometer, which was used to analyze the influence of surface textures on the frictional shear stress is given. To do so, a long frictional path has to be analyzed and ensure that an untreated surface texture is in contact with the pin along the frictional path. The experimental results are further complemented by the development of a numerical FE-model to analyze the influence of different surface textures on the friction shear stress. These results a correlated with shot peening parameters of the peening process to distinguish a parameter set to reduce the frictional shear stress the most for each shot peening medium. After the first step of tribometer tests, the surface textures are applied on specimens for an extrusion process in order to distinguish the most suitable surface texture to reduce friction and by that the punch force.

\subsection{Deposition process of the $(\mathrm{Cr}, \mathrm{Al}) \mathrm{N}+\mathrm{Mo}: \mathrm{S}$ coating}

The $(\mathrm{Cr}, \mathrm{Al}) \mathrm{N}+\mathrm{Mo}$ :S-coatings were deposited by means of the hybrid direct current magnetron sputtering/high power pulsed magnetron sputtering (dcMS/HPPMS) technology in an industrial scale coating unit CC800/9 Custom, CemeCon AG, Wuerselen, Germany. The unit is constructed with a chamber volume of $\mathrm{V}=1 \mathrm{~m}^{3}$. For the deposition five CrAl20 (Cr-base plate with $20 \mathrm{Al}$ plugs) with a purity of $\mathrm{w}_{\mathrm{Cr}}=99.9$ wt. $-\%$, $\mathrm{w}_{\mathrm{Al}}=99.5$ wt. $-\%$ and one $\mathrm{MoS}_{2}$ target with a purity of $\mathrm{w}_{\mathrm{MoS}_{2}}=99.5$ wt. $\%$ were used. The $\mathrm{MoS}_{2}$ target was mounted on one of the four dcMS cathode. In order to ensure a sufficiently high adhesion strength between the top layer and the substrate, a $(\mathrm{Cr}, \mathrm{Al})$ bond coat and a $(\mathrm{Cr}, \mathrm{Al}) \mathrm{N}$ interlayer were deposited first. The process parameters for the deposited coatings are listed in Table 1. The process parameters of the $(\mathrm{Cr}, \mathrm{Al}) \mathrm{N}+\mathrm{Mo}: \mathrm{S}$ top layer are explained in more detail in Bobzin et al. [19].

Table 1. Process parameters for deposition of the $(\mathrm{Cr}, \mathrm{Al}) \mathrm{N}+\mathrm{Mo}$ :S-coatings

\begin{tabular}{|l|c|c|}
\hline \multicolumn{1}{|c|}{ Process parameter } & Unit & \\
\hline Depostion time, $\mathrm{t}$ & $\mathrm{Min}$ & 107 \\
\hline Argon flux, $\mathrm{j}(\mathrm{Ar})$ & $\mathrm{sccm}$ & 200 \\
\hline Nitrogen flux, $\mathrm{j}\left(\mathrm{N}_{2}\right)$ & $\mathrm{sccm}$ & $\begin{array}{c}\text { Pressure } \\
\text { controlled }\end{array}$ \\
\hline Pressure, $\mathrm{p}$ & $\mathrm{mPa}$ & 710 \\
\hline dcMs cathode power, $\mathrm{P}_{\text {dcMS-Mos2 }}$ & $\mathrm{kW}$ & 2 \\
\hline dcMs cathode power, $\mathrm{P}_{\text {dcMS-CrAl20 }}$ & $\mathrm{kW}$ & 3 \\
\hline HPPMS mean cathode power, $\mathrm{P}_{\text {HPPMS-CrA120 }}$ & $\mathrm{kW}$ & 5 \\
\hline Pulse length, $\mathrm{t}_{\text {on }}$ & $\mathrm{\mu s}$ & 40 \\
\hline Frequency & $\mathrm{Hz}$ & 500 \\
\hline Bias voltage, $\mathrm{U}_{\text {Bias }}$ & $\mathrm{V}$ & 100 \\
\hline
\end{tabular}

\subsection{Analysis of the influence of shot peening parameters on evolving surface textures}

Shot peening by steel casks is commonly used as workpiece preparation in industrial cold forging processes [13]. The surface modification supports a friction reduction in combination with the use of a lubricant. In dry metal forming, as a result of the missing lubricant a higher friction is expected. In order to get the most suitable surface texture for a friction reduction in cold 
forging, different peening media were investigated [13]. Besides steel casks, ceramic beads and corundum particles were used with different peening parameters, Table 2.

Table 2. Shot peening parameters in dependency of shot media, $($ Steel $=\mathrm{St}$, Ceramic $=\mathrm{Ce}$, Corundum $=\mathrm{Co})$

\begin{tabular}{|c|c|c|c|c|}
\hline Medium & $\begin{array}{c}\text { Particle } \\
\text { form }\end{array}$ & $\begin{array}{c}\text { Particle } \\
\text { size [um] }\end{array}$ & $\begin{array}{c}\text { Density } \\
{\left[\mathbf{g} / \mathbf{c m}^{\mathbf{3}}\right]}\end{array}$ & $\begin{array}{c}\text { Hardness } \\
{[\mathrm{HV}]}\end{array}$ \\
\hline $\mathrm{St}$ & round & $700-1,000$ & 7.8 & $390-535$ \\
\hline $\mathrm{Ce}$ & round & $125-250$ & 3.8 & $\sim 1,200$ \\
\hline $\mathrm{Co}$ & sharp & $425-600$ & $3.9-4.1$ & $\sim 2,600$ \\
\hline
\end{tabular}

The best peening parameters for a reduction of the fricitonal shear stress in tribometer tests are the ones shown in Table 3 [14]. Furthermore, a rather sharp and hard surface texture as the one shot by corundum particles, reduced the frictional shear stress the most.

Table 3. Resulting surface integrity after shot peening $($ Steel $=\mathrm{St}$, Ceramic $=\mathrm{Ce}$, Corundum $=\mathrm{Co}$, Ref $=$ Reference $)$

\begin{tabular}{|c|c|c|c|c|c|}
\hline \multirow{2}{*}{ Surface } & \multicolumn{2}{|c|}{ Peening parameters } & \multicolumn{3}{c|}{ Surface integrity } \\
\cline { 2 - 6 } & $\mathrm{p}[\mathrm{bar}]$ & $0[\%]$ & HV10 [HV] & Sa [um] & Sz [um] \\
\hline St & 3 & 100 & 280 & 2.23 & 25.7 \\
\hline Ce & 2 & 100 & 410 & 1.38 & 26.8 \\
\hline Co & 4 & 100 & 260 & 4.09 & 59.7 \\
\hline Ref & & & 200 & 1.63 & 29.5 \\
\hline
\end{tabular}

\subsection{Development and Use of a Pin-on-Cylinder Tribometer}

For an explicit investigation of the influence of the surface textures on the frictional shear stress conventional tribometers as a pin-on-disc-tribometer cannot be used since textures are flattened after the first revolution of the disc. To analyze the surface textures intensively, a new frictional path per revolution has to be used. Thus, a pinon-cylinder (POC) tribometer concept was realized on a lathe, see Fig. 2.
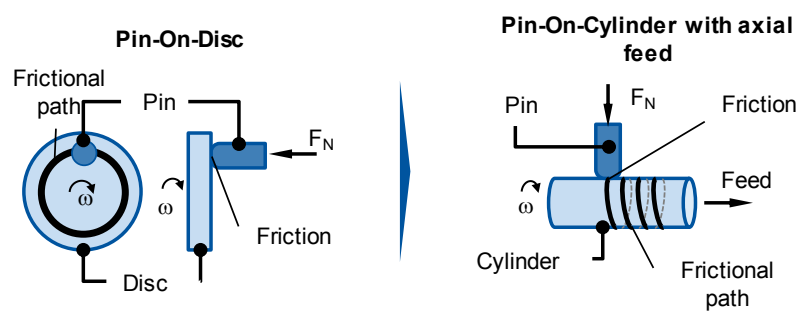

Fig. 2. Schematic overview of Pin-On-Cylinder Tribometer

The normal load is applied by means of a hydraulic actuator [15]. Due to these investigations, results regarding the influence of the contact between selflubricating coatings and surface textures on the friction shear stress were obtained [18]. Shot peening by corundum particles as the best treatment to reach lower frictional shear stresses in this interaction was confirmed, see Fig. 3.

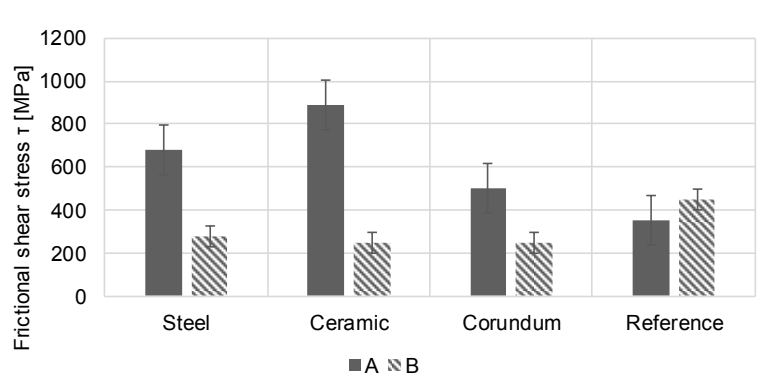

Fig. 3. Frictional shear stress during tribometer tests with uncoated (A) and coated (B) tools

\subsection{Effect of surface textures on the punch force in an extrusion process}

After investigating surface textures using tribometer tests, the surface textures were applied in an extrusion process [16]. As in the tribometer experiments, the named surface textures were used. As coating a slighty adapted one compared to the tribometer tests were used. The punch velocity was a constant $v_{\mathrm{P}}=5 \mathrm{~mm} / \mathrm{s}$ and the punch force was measured throughout the whole process. The punch force in extrusion processes is greatly influenced by the friction [17]. A lower punch force corresponds to a lower friction during the process. The results showed, that the lowest punch force was achieved from the combination of ceramic shot peened surface texture with self-lubricating tool coating, Fig. 4.

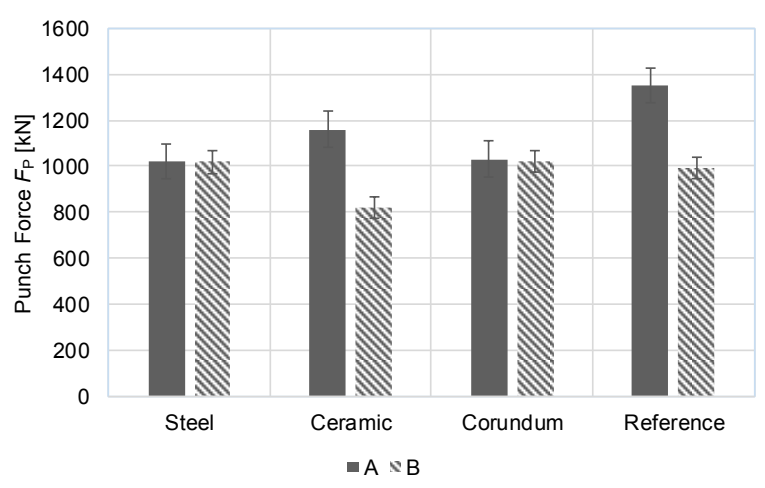

Fig. 4. Maximum punch forces of workpieces with different surface structures and uncoated (A) and coated (B) tools

The comparison between the results of the tribometer tests and the extrusion process show a discrepancy. A hard and rough surface structure results in low frictional shear stresses in the tribometer tests, whereas a softer and slightly rough surface texture leads to lower punch forces in the extrusion processes. This lack of knowledge needs to be overcome. To do so, smoothing effects for each surface texture need to be investigated. In dependency of the contact normal force, smoothing of the different surface textures manufactured by shot peening with different media is researched.

\section{Experimental set-up}

This section describes both the testing of the specimens and the evaluation of the surface textures using 
roughness and hardness measurements. As specimen material the quenched and tempered steel 42CrMo4 (DIN: 1.7225, AISI: 4140) was used. This steel is used for highly loaded parts in automotive or aircraft industries, e.g. gears, spindles, rods, and rams. Therefore, the material is very important for the forming industry. As a tool material the cold working tool steel X155CrMoV12 (DIN: 1.2379, AISI D2) was used for the pin. It is a typical tool material in cold forging [17]. X155CrMoV12 was hardened to $62 \mathrm{HRC}$ and the tool surfaces were polished $(\mathrm{Rz}=1)$ according to the industrial standard. The experiments were conducted using the Pin-on-Cylinder Tribometer, without any revolution, see Fig. 5.

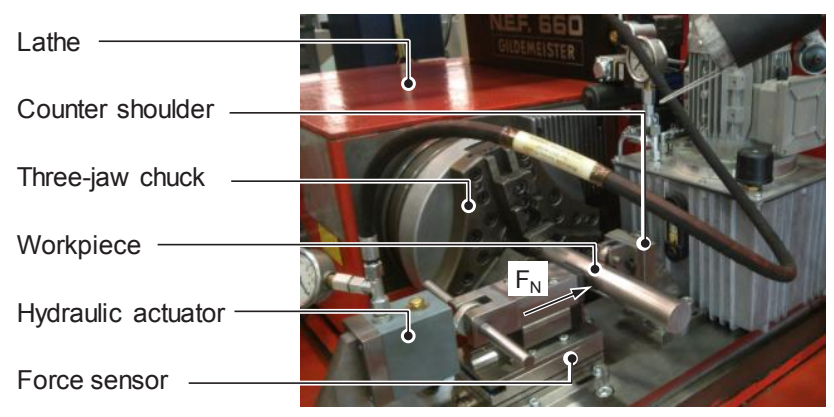

Fig 5. Pin-On-Cylinder Tribometer with different active parts

In order to analyze the impact of different surface textures during the smoothing process, different normal contact forces were applied. Before and afterwards the experiments, the surface roughness and hardness of the specimens were analyzed. For the experiments a turning lathe was used and the contact pressure was applied by means of a hydraulic actuator. Five different normal contact forces were applied: $F_{\mathrm{N}}=[460 ; 640 ; 1180 ; 1850$; $2600 \mathrm{~N}]$. Each experiment was repeated three times to account for statistical effects.

\section{Surface properties}

The surface hardness was quantified by means of Vickers hardness according to DIN EN ISO 6507 and ASTM E384 using a Wilson Universal Hardness Tester UH250. 16 measurements using a testing load of $30 \mathrm{~N}$ were performed on polished cross-sections of each specimen. The 16 measurements were then averaged.

The surface roughness was measured using a combined roughness and contour measurement system Hommel Etamic nanoscan 855 made by Jenoptik AG, Germany. According to preliminary work, the surface parameters Sa and Sz were evaluated based on standard to ISO 25178 and EUR $15178 \mathrm{~N}$ in a measurement grid of $5 \times 5 \mathrm{~mm}$. A contact tip with a tip angle of 60 degrees and a tip radius of $2 \mu \mathrm{m}$ was used. Sa describes the averaged roughness of a surface, Sz its maximum height.

Furthermore, laser scanning microscopy analysis was conducted in order to measure the size of each indentation. Laser scanning microscope Keyence VK$\mathrm{X} 100$ was used. Each indentation has a specific size. In order to get the maximum resolution, for each indentation a specific measuring area was selected.

\section{Results and Discussion}

The reference specimen (Ref) represents the initial state of the workpiece after the turning process and before any shot peening or other machining process, see Fig. 6.
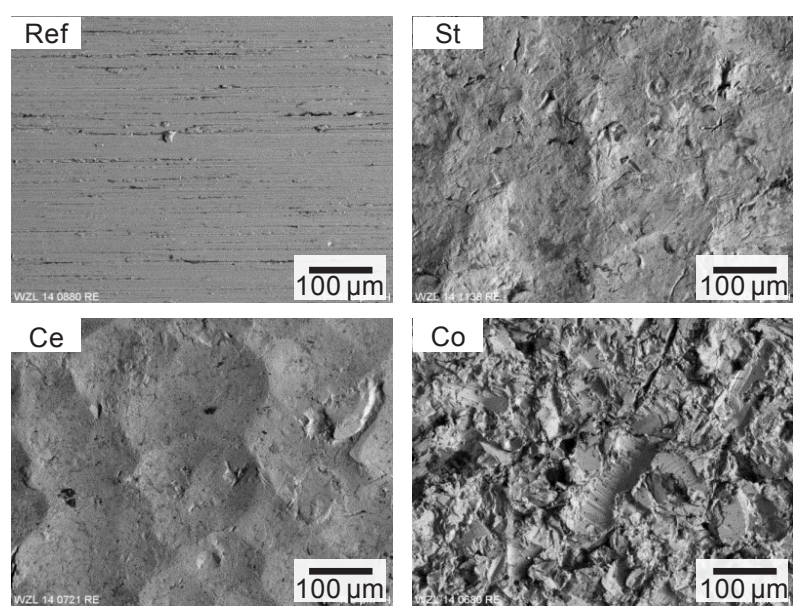

Fig 6. Surface textures before machining (Steel $=\mathrm{St}$, Ceramic $=\mathrm{Ce}$, Corundum $=\mathrm{Co}, \mathrm{Ref}=$ Reference $)$

The other specimens are shot peened by steel casks $(\mathrm{St})$, ceramic casks $(\mathrm{Ce})$, and by corundum particles $(\mathrm{Co})$. Scanning electron microscopy images (SEM) show, that the reference structure is even. The steel and ceramic shot peened specimens have some craters, but remain in a flat surface. The corundum shot peened surface texture has many craters and is rough at the same time.

After indentation tests were done, laser scanning microscopy analysis were made. The results for a normal contact force of $F_{\mathrm{N}}=2,600 \mathrm{~N}$ show the indentation clearly, see Fig. 7. The indentations were measured according to every specimen and contact normal force. This indicates, which surface texture has the biggest resistance against the plastic deformation. The elliptical extent was measured and the area of the ellipsoid was calculated via:

$$
A=\pi \cdot w \cdot h
$$

$A$ is the area, $w$ is the width and $h$ the height of the contact ellipse. In this example, The indentation on the ceramic shot peened surface texture resulted in an area $A_{\mathrm{Ce}}=4.7 \mathrm{~mm}^{2}$, the steel shot peened one in $A_{\mathrm{St}}=6.6 \mathrm{~mm}^{2}$, the corundum shot peened one $A_{\mathrm{Co}}=7.1 \mathrm{~mm}^{2}$, and the reference surface texture in $A_{\text {Ref }}=8.3 \mathrm{~mm}^{2}$. Each experiment was repeated three times to account for statistical effects. 

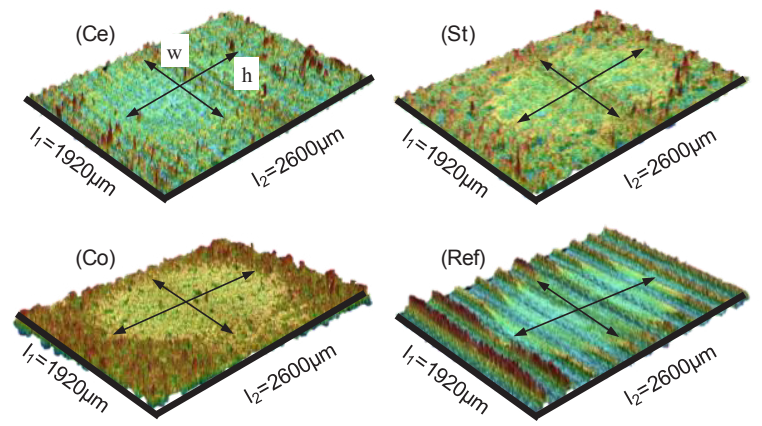

Fig 7. Laser Scanning Microscopy pictures with indentation by a normal force of $F_{\mathrm{N}}=2,600 \mathrm{~N}$ and their resulting area $($ Steel $=$ St, Ceramic $=\mathrm{Ce}$, Corundum $=\mathrm{Co}$, Ref $=$ Reference $)$

Table 4. Extent of indentation area generated by a forming force of $\mathrm{FN}=2,600 \mathrm{~N}($ Steel $=\mathrm{St}$, Ceramic $=\mathrm{Ce}$,

Corundum $=\mathrm{Co}$, Ref $=$ Reference)

\begin{tabular}{|c|c|c|c|}
\hline Medium & $w=$ width [um] & $\boldsymbol{h}=\boldsymbol{h e i g h t}[\boldsymbol{\mu m}]$ & $\boldsymbol{A}=$ Area $\left[\mathrm{mm}^{2}\right]$ \\
\hline Ce & 809 & 1,344 & 4.7 \\
\hline St & 1,108 & 1,730 & 6.6 \\
\hline Co & 961 & 1,772 & 7.1 \\
\hline Ref & 1,299 & 1,827 & 8.3 \\
\hline
\end{tabular}

The evaluation of all indentations shows that due to a higher contact normal force greater indentation areas evolve, see Table 4 . The rise of the area is not linear. The reason for these irregularities is the varying surface roughness on each specimen. The smoothing mechanism starts with a contact of the tool with the roughness peaks. In the beginning, there is a plastic deformation on a microscopic level. The more the roughness peaks are smoothed in, the more material gets into contact with the tool and a plastic deformation on a macroscopic level proceeds. Depending on the section, some higher roughness peaks are smoothed in faster than others and the plastic deformation on a macroscopic level proceeds earlier. Overall, the indentations on the reference surface texture evolve into the greatest indentations. The reference surface structure was not pre-treated, so no strain hardening took place due to surface modifications. The roughness of the surface was low, so from the beginning on, nearly the whole surface was in contact with the tool. The surface texture shot peened by ceramic casks resulted in the smallest indentation areas. The surface texture is a bit rougher than the reference structure in the beginning, but not as rough as the steel or corundum shot peened surface texture. At the same time it is the hardest surface texture. Due to a higher roughness and a higher hardness than the reference surface texture, the smallest indentation area evolve. The ceramic shot peened specimen has a lower roughness than the other peened specimens have. Due to the lower roughness, more material gets into contact with the pin from the beginning on. Therefore, the contact normal force results in a lower contact normal pressure, as in a rougher surface texture, which results in the lower indentation area. The surface roughness is the dominant criteria for the evolution of the indentation area in the beginning of the process. When the forces rise the strain hardening due to the pretreatment becomes the dominant criterion and supports a lower plastic deformation. In combination with the results of the extrusion process, a surface texture, which results in a smaller indentation area is the most suitable one to lower punch forces and friction in extrusion at the same time.

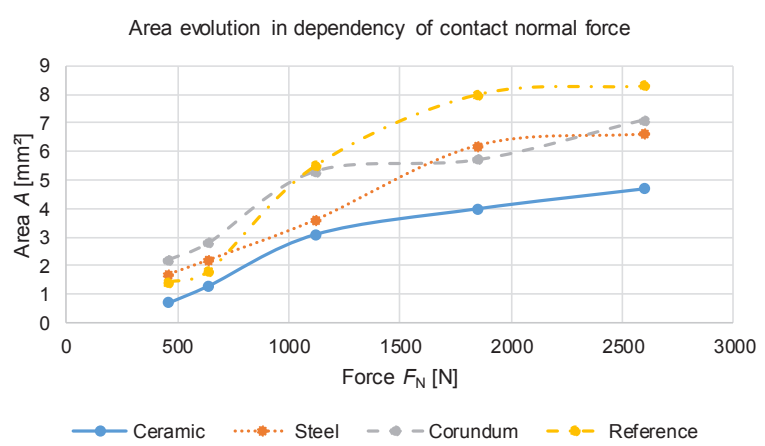

Fig. 8. Indentation area in dependency of shot peening medium and contact normal force

\section{Summary and Outlook}

The resulting discrepancy between the frictional shear stress during tribometer testing and the punch force in an extrusion process led to an investigation of the plastic deformation of different surface textures. A reference surface texture machined by turning as well as several shot peened specimens, peened by steel, and ceramic casks and by corundum particles were investigated. Due to different pretreatments, different surface integrities evolved. These surface textures were tested with increasing normal contact forces. Rising contact normal forces resulted in larger indentation areas. Furthermore, a harder and rather flat surface texture resulted in the smallest indentation area, whereas a flat but soft surface texture resulted in the greatest indentation area. Respective to the lowest punch force, an ideal workpiece has a hard surface texture with a low roughness. In order to separate the effects of roughness and hardness of the surface texture, further researches have to be done. Hardened and unhardened with an identic roughness as well as rougher or flatter surface textures with an identic hardness need to be investigated.

\section{Acknowledgments}

The research was funded by the German Research Foundation (Deutsche Forschungsgemeinschaft DFG) within the priority program "Dry metal forming sustainable production through dry processing in metal forming" (SPP 1676). The authors also thank Presswerk Krefeld $\mathrm{GmbH}$ for their support supplying tools and workpieces as well as conducting the full forward extrusion process.

\section{References}

1. N. Bay, A. Azushima, P. Groche, I. Ishibashi, M. Merklein, M. Morishita, T. Nakamura, S. Schmid, M. Yoshida: Environmentally benign tribo-systems for metal forming. In: Annals of the CIRP 59/2 (2010) 760-780 
2. F. Vollertsen, F. Schmidt: Dry Metal Forming: Definition, Chances and Challenges. In: Int. J. Precision Engineering and Manufacturing -Green Technology 1/1 (2014) 59-62

3. Vollertsen, F. and Schmidt, F. (2014). Dry metal forming: Definition, Chances and Challenges. Int $J$ Precis Eng Man - Green Technology 1(1):59-62

4. K. Müller (2003) Praktische Oberflächentechnik Vorbehandeln - beschichten - Beschichtungsfehler - Umweltschutz; JOT Fachbuch

5. M. Teller, M. Bambach, G. Hirt: A compressiontorsion-wear-test achieving contact pressures of up to eight timest he initial flow stress of soft aluminium. In: CIRP Annals - Manufacturing Technology 64 (2015) 280-292

6. M. Teller, M. Bambach, G. Hirt, I. Ross, A. Temmler, R. Poprawe, H. Bolvardi, S. Prünte, J. M. Schneider: Investigation of the suitability of surface treatments for dry cold extrusion process-oriented tribological testing. In: Key Engineering Materials Vols. 651-653 (2015) 473-479

7. M. Murakawa, N. Koga, T. Kumagai: Deep-drawing of aluminum sheets without lubricant by use of diamond-like carbon coated dies. Surf Coat Tech 76 (1995) 553-558

8. M. Murakawa, S. Takeuchi: Evaluation of tribological properties of DLC films used in sheet forming of aluminum sheet. Surf Coat Tech 163 (2003) 561-565.

9. K. Osakada R. Matsumoto: Fundamental Study of Dry Metal Forming with Coated Tools. CIRP Ann. Manuf. Technol 49/1 (2000) 161-164.

10. S. Kataoka, A. Motoi. Improvement in DLC thin film adhesion and its application to dry deep drawing. J. Jpn. Soc. Technol. Plast 46/532 (2005) 412-416.

11. K. Tamaoki, S. Kataoka: Study of deep drawing using diamond coated tools. Journal of Material Testing Research Assoc. of Japan 53/4 (2008) 247253.

12. K. Tamaoki, S. Kataoka, K. Minamoto: Dry deepdrawing with use of electroconductive ceramic tools. Proc. Int. Conf. Trib. Manuf. Pro (2007) 175-179.

13. Z. G. Wang, S. Komiyama, Y. Yoshikawa, T. Suzuki, K. Osakada (2015) Evauluation of lubricants without zinc phosphate precoat in multistage cold forging, In: CIRP Annals Manufacturing Technology 64

14. P. Groche, J. Stahlmann, J. Hartel, M. Köhler (2009) Hydrodynamic effects of macroscopic deterministic surface structures in cold forging processes, In: Tribology International 42

15. Klocke, F., Trauth, D., Schongen F. and Shirobokov, A. (2014). "Analysis of friction between stainless steel sheets and machined hammer peened structured tool surfaces." Prod. Eng. Res. Devel. 8: 263-272.

16. Bobzin, K., Brögelmann, T., Bastürk, S., Klocke, F., Mattfeld, P., Trauth, D. (2015). "Development of an insitu Plasma Treatment of X155CrMoV12 for a (Cr,Al)N PVD Tool Coating for Dry Metal Forming in Cold Forging." Dry Metal Forming Open Access Journal 1(1): 57-62.

17. Bobzin, K., Brögelmann, N.C., Bastürk, S., Klocke, F., Mattfeld, P., Trauth, D. (2015). "Tribological Behavior of $\left(\mathrm{Crl}_{-\mathrm{x}} \mathrm{Al}_{\mathrm{x}}\right) \mathrm{N} / \mathrm{WS}_{\mathrm{y}}$ PVD Tool Coatings for the Application in Dry Cold Forging of Steel." Dry Metal Forming Open Access Journal (1)1:152158.

18. Trauth, D.; Hild, R.; Mattfeld, P.; Bastürk, S.; Brögelmann, T.; Bobzin, K.; Klocke, F.: Advances in dry metal forming of low alloyed steels for cold forging using a $(\mathrm{Cr}, \mathrm{Al}) \mathrm{N}$ tool coating and surface structures on workpieces. In: Proceedings of the 12th International Conference THe „A“ coatings in Manufacturing Engineering, Hannover, Germany, March 31st - April 1st (2016)

19. Bobzin, K., Kruppe, N.C., Arghavani, M, Hoffmann, D.C., Klocke, F., Mattfeld, P., Trauth, D., Hild R. (2017). "Mechanical and tribological characterization of self-lubricating $\left(\mathrm{Cr} 1-_{\mathrm{x}} \mathrm{Al} \mathrm{l}_{\mathrm{x}}\right) \mathrm{N}$ coatings for deposition on complex-shaped forging toolsMechanical and tribological characterization of self-lubricating $\left(\mathrm{Cr} 1-_{\mathrm{x}} \mathrm{Al}_{\mathrm{x}}\right) \mathrm{N}$ coatings for deposition on complex-shaped forging tools." Dry Metal Forming Open Access Journal (3) 81-89. 